\title{
Insights into the three-dimensional Lagrangian geometry of the Antarctic polar vortex
}

\author{
Jezabel Curbelo ${ }^{1,2}$, Víctor José García-Garrido ${ }^{1}$, Carlos Roberto Mechoso ${ }^{3}$, Ana Maria Mancho ${ }^{1}$, Stephen Wiggins ${ }^{4}$, \\ and Coumba Niang ${ }^{1,5}$ \\ ${ }^{1}$ Instituto de Ciencias Matemáticas, CSIC-UAM-UC3M-UCM. C/ Nicolás Cabrera 15, Campus de Cantoblanco UAM, \\ 28049 Madrid, Spain \\ ${ }^{2}$ Departamento de Matemáticas, Facultad de Ciencias, Universidad Autonóma de Madrid, 28049 Madrid, Spain \\ ${ }^{3}$ Department of Atmospheric and Oceanic Sciences, University of California at Los Angeles, Los Angeles, California, USA \\ ${ }^{4}$ School of Mathematics, University of Bristol, Bristol BS8 1TW, UK \\ ${ }^{5}$ Laboratoire de Physique de l'Atmosphere et de l'Ocean Simeon Fongang, Ecole Superieure Polytechnique, \\ Universite Cheikh Anta Diop, 5085, Dakar-Fann, Senegal
}

Correspondence to: Ana Maria Mancho (a.m.mancho@icmat.es)

Received: 10 February 2017 - Discussion started: 16 February 2017

Revised: 8 June 2017 - Accepted: 16 June 2017 - Published: 25 July 2017

\begin{abstract}
In this paper we study the three-dimensional (3-D) Lagrangian structures in the stratospheric polar vortex (SPV) above Antarctica. We analyse and visualize these structures using Lagrangian descriptor function $M$. The procedure for calculation with reanalysis data is explained. Benchmarks are computed and analysed that allow us to compare 2-D and 3D aspects of Lagrangian transport. Dynamical systems concepts appropriate to 3-D, such as normally hyperbolic invariant curves, are discussed and applied. In order to illustrate our approach we select an interval of time in which the SPV is relatively undisturbed (August 1979) and an interval of rapid SPV changes (October 1979). Our results provide new insights into the Lagrangian structure of the vertical extension of the stratospheric polar vortex and its evolution. Our results also show complex Lagrangian patterns indicative of strong mixing processes in the upper troposphere and lower stratosphere. Finally, during the transition to summer in the late spring, we illustrate the vertical structure of two counterrotating vortices, one the polar and the other an emerging one, and the invariant separatrix that divides them.
\end{abstract}

\section{Introduction}

Over the past several decades the mathematical theory of dynamical systems has provided a fruitful framework to describe the transport and mixing processes that take place in fluids and to understand the underlying flow structures associated with these phenomena. The seminal paper by Aref (1984) on chaotic advection sparked interest in this perspective, which is inspired by the work of Poincaré. For the understanding of particle dynamics, Poincaré sought a geometrical approach that was based on geometrical structures and their role in organizing all trajectories into regions corresponding to qualitatively different dynamical fates. These structures have been referred to as Lagrangian coherent structures (LCS) in the fluid mechanics community (Haller and Yuan, 2000; Shadden et al., 2005).

Many studies of LCS in the atmosphere and in the ocean have been performed in a two-dimensional (2-D) scenario. This is because in an appropriate range of space scales and timescales a Lagrangian property of the particles is approximately unchanged in time. Hence, the flow can be assumed to occur on surfaces on which that property is constant. For instance, stratospheric flows on the timescale of stratospheric sudden warmings ( $\sim 10$ days) are, to a first approximation, adiabatic and frictionless, and thus fluid particles and their trajectories are constrained to remain on surfaces of constant 
specific potential temperature (isentropic surfaces). Bowman (1993) and Joseph and Legras (2002) have examined transport processes across the Antarctic stratospheric polar vortex (SPV) on isentropic surfaces, which are quasi-horizontal in the atmosphere. Also, for oceanic flows it is often assumed that fluid parcels remain on surfaces of constant density (isopycnals), which are quasi-horizontal. Mancho et al. (2006), d'Ovidio et al. (2009) and Branicki et al. (2011) followed the isopycnal approach for oceanic applications in the Mediterranean Sea, Mendoza et al. (2014) for the Gulf of Mexico and García-Garrido et al. $(2015,2016)$ for other ocean areas.

Geophysical flows, however, are not 2-D. The study of transport processes in 3-D flows brings into the discussion issues about the three-dimensional (3-D) visualization of Lagrangian structures (see e.g. Wiggins, 2010). In idealized 3D time-dependent flows Poincaré sections have been used to recognize significant Lagrangian structures (Cartwright et al., 1996; Pouransari et al., 2010; Moharana et al., 2013; Rypina et al., 2015). Invariant manifolds acting as transport barriers in 3-D flows may have the structure of convoluted 2-D surfaces embedded in a volume (Branicki and Wiggins, 2009). In oceanic contexts these surfaces have been identified by stitching together 2-D Lagrangian structures in different layers (Branicki and Kirwan, 2010) or connecting ridges computed from finite-size Lyapunov exponent fields (Bettencourt et al., 2014). In the field of atmospheric sciences, these structures have similarly been obtained by connecting ridges computed from finite-time Lyapunov exponents (FTLE) (Rutherford and Dangelmayr, 2010; du Toit and Marsden, 2010; Lekien and Ross, 2010). More recently, also in atmospheric contexts, 3-D Lagrangian information has been extracted by means of 2-D slices of the full 3D FTLE field computed from 3-D trajectories (Rutherford et al., 2012).

The methodology used in this paper for visualizing 3-D Lagrangian structures in the stratosphere is based on the Lagrangian descriptor (LD) known as the $M$ function (Madrid and Mancho, 2009; Mendoza and Mancho, 2010; Mancho et al., 2013). So far, in the stratosphere context, the $M$ function has been used to gain insight into key dynamical and transport processes in 2-D settings (de la Cámara et al., 2012, 2013; Smith and McDonald, 2014; Guha et al., 2016; Manney and Lawrence, 2016; García-Garrido et al., 2017). More recently, Mancho et al. (2013) and Lopesino et al. (2017) have applied the $M$ function to the visualization of structures in idealized 3-D flows. Rempel et al. (2013) have applied the $M$ function to visualize coherent structures in full 3-D direct numerical simulations of the compressible magnetohydrodynamic equations. The $M$ function has the advantage of highlighting simultaneously invariant manifolds by means of singular features and also tori-like coherent structures (see Mendoza and Mancho, 2010; de la Cámara et al., 2012; Rempel et al., 2013; Mezic and Wiggins, 1999; Lopesino et al., 2017). Here we apply $M$ to produce a full 3-D description from 3-D flows above Antarctica during a period in the spring of 1979 in which the stratosphere was both rather stable (August) and subjected to rapid changes (October) (Yamazaki and Mechoso, 1985). In this region, the later period selected for analysis comprises an interval when the winter circulation, which is characterized by a strong circumpolar westerly (cyclonic) flow known as the SPV, breaks down as the final warming to summer conditions develops. Although final warmings in the Southern Hemisphere are broadly similar each year (Mechoso et al., 1988), they can be punctuated by periods of rapid changes. During the final warming of 1979 , the transition from winter to summer circulation accelerated during mid-October, a period when perturbing waves were very active and the vertical energy flux from the troposphere intensified (Yamazaki and Mechoso, 1985). Our aim is to describe and visualize these phenomena from a full 3-D perspective using reanalysis data. To our knowledge, this is the first time that the potential of $M$ to achieve this goal is explored.

The article is organized as follows. Section 2 describes the dynamical systems approach to the analysis of 3-D Lagrangian structures. Section 3 describes the dataset used in this study, the calculation of $M$ in 3-D, the data postprocessing needed to this end, the computational procedures and other issues involved in this task. Section 4 discusses some benchmark calculations and their interpretation. Section 5 provides the results and findings of $M$ on the 3-D Lagrangian structure of the polar vortex on August and in mid-October 1979. Finally, Sect. 6 includes a discussion and presents our conclusions.

\section{The dynamical systems approach to the analysis of 3-D Lagrangian structures}

The theory of dynamical systems provides an ideal framework for studying nonlinear transport and mixing processes in the atmosphere. The geometrical structures that vertebrate the Lagrangian skeleton act as material barriers that fluid particles cannot cross. A key element in the dynamical description is the presence of hyperbolic regions defined by rapid fluid contracting and expanding rates along directions that are respectively associated with the stable and unstable manifolds. In 2-D flows, these manifolds are curves, while in 3-D settings, other possibilities arise. We discuss some particularities for the system under study next.

If we assume that air parcels are passively advected by the flow, the dynamical system that governs the atmospheric flow is given by

$\dot{\mathbf{x}}=\mathbf{v}(\mathbf{x}(t), t), \quad \mathbf{x}\left(t_{0}\right)=\mathbf{x}_{0}$,

where $\mathbf{x}\left(t ; \mathbf{x}_{0}\right)$ represents the trajectory of an air parcel that at time $t_{0}$ is at position $\mathbf{x}_{0}$, and $\mathbf{v}$ is the velocity field. For the geophysical context we are focusing on, the velocity components will be supplied by the ERA-Interim Reanalysis 
dataset produced at the European Centre for Medium-Range Weather Forecasts, as explained in detail in the next section. This dataset confirms that the magnitude of the vertical velocity component in the stratosphere is very small, so that vertical displacements of fluid parcels compared to the horizontal displacements are also small for the timescales of interest in this study ( $\sim$ days). These considerations motivate the discussion of a system with the particular structure given in Eq. (2) as it will support the interpretation of the findings described in Sect. 5 in this region of the atmosphere.

For two-dimensional flows hyperbolic trajectories and their stable and unstable manifolds are the key kinematical features responsible for the geometrical template governing transport. However, in three dimensions there are new types of three-dimensional structures that may form a geometrical template that governs transport. The "weak threedimensionality" of many geophysical flows, like the one considered in this paper, gives rise to a (normally) hyperbolic invariant curve (i.e. not a single trajectory) that has twodimensional stable and unstable manifolds embedded in the 3-D space. In this case the stable and unstable manifolds of this invariant curve are codimension one in the flow and therefore provide barriers to transport. Moreover, since in this case the stable and unstable manifolds are both codimension one, they can intersect to form lobes, resulting in a three-dimensional version of lobe dynamics. We now describe the special form of the flow giving rise to this structure. The form of the flow follows from Wiggins (1988) and was described in the context of fluid mechanics in Mezić and Wiggins (1994).

Here we examine a simple model three-dimensional velocity field that captures the form of the velocity field given by the dataset that we study and, therefore, allows us to describe this less familiar notion of a normally hyperbolic invariant curve in a simple setting. The velocity field has the following form:

$$
\left\{\begin{array}{l}
\frac{\mathrm{d} x}{\mathrm{~d} t}=\frac{\partial H(x, y, z, t)}{\partial y}=v_{x}(x, y, z, t) \\
\frac{\mathrm{d} y}{\mathrm{~d} t}=-\frac{\partial H(x, y, z, t)}{\partial x}=v_{y}(x, y, z, t) \\
\frac{\mathrm{d} z}{\mathrm{~d} t}=0
\end{array}\right.
$$

where $H(x, y, x, t)=A(z) \sin (\pi y) \sin (\pi x) / \pi$ and $A(z)=$ $1+\sin (\pi z / 2)$. The system is defined in the domain $(x, y, z) \in$ $[0,1] \times[-1,1] \times[-1,1]$. We refer to $x$ and $y$ as the horizontal coordinates and to $z$ as the vertical coordinate. This velocity field is certainly "weakly" three-dimensional as there is no motion in the vertical direction, yet the horizontal motion does depend on the height. This model contains the essence of the geometrical structures governing transport in the dataset that we analyse.

More specifically, note that for each $z$ the system (2) has a hyperbolic fixed point at $(x=0, y=0)$, for which the lin- earized system is

$$
\begin{aligned}
& \frac{\mathrm{d} x}{\mathrm{~d} t}=A(z) \pi x, \\
& \frac{\mathrm{d} y}{\mathrm{~d} t}=-A(z) \pi y .
\end{aligned}
$$

The curve $(0,0, z)$ is clearly an invariant curve (in particular, it is a curve of fixed points). The linearized stability described by Eqs. (3) and (4) quantifies linearized stability normal to the invariant curve $(0,0, z)$ and, hence, is the origin of the phrase normal hyperbolicity. For each $z$, the fixed point has a one-dimensional stable manifold and a one-dimensional unstable manifold. Therefore as a function of $z$ the curve $(0,0, z)$ has two-dimensional stable and two-dimensional unstable manifolds in three dimensions. Hence, these twodimensional invariant surfaces provide barriers to transport in the three-dimensional flow (Wiggins, 1988; Mezić and Wiggins, 1994). The geometrical representation of the manifolds is shown in Fig. 1a.

The 3-D Lagrangian structure of Eq. (2) visualized in Fig. $1 \mathrm{~b}$ is achieved by means of the $M$ function, which is represented on slices intersecting the manifolds. Other slice choices are possible, but here we just show two simple possibilities on perpendicular planes which help to capture the essential features of the full 3-D motion. The $M$ function is defined as follows:

$$
M\left(\mathbf{x}_{0}, t_{0}, \tau\right)=\int_{t_{0}-\tau}^{t_{0}+\tau}\left\|\mathbf{v}\left(\mathbf{x}\left(t ; \mathbf{x}_{0}\right), t\right)\right\| \mathrm{d} t
$$

where $\mathbf{v}(\mathbf{x}, t)$ is the velocity field and $\|\cdot\|$ denotes the Euclidean norm. At a given time $t_{0}, M$ corresponds to the length of the trajectory traced by a fluid parcel starting at $\mathbf{x}_{0}=\mathbf{x}\left(t_{0}\right)$ as it evolves forwards and backwards in time for a time interval $\tau$. For sufficiently large $\tau$ values the sharp changes that occur in narrow gaps in the scalar field provided by $M$, which we will refer to as singular features, highlight the stable and unstable manifolds and, at their crossings, hyperbolic trajectories as confirmed by Fig. 1b. Recently, Lopesino et al. (2017) have established a rigorous mathematical foundation for specific LDs for a class of examples in continuous timedynamical systems.

Figure $1 \mathrm{~b}$ shows that the $M$ function also has the capability of revealing vortices present in the fluid. In particular for this example two counterrotating vortices which are vertically extended are visible. The yellowish colours highlight the parts of the vortices with the highest speeds. Typically vortex- or jet-like structures (for periodic domains) are related to 2-tori in three-dimensional flows. This is discussed in Mezić and Wiggins (1994) and Wiggins (2010). This notion is related to fluid regions trapping fluid parcels in their interior and isolating them from the surrounding fluid, as for instance is the case for the circulating strong jet forming the SPV. There exist formal results linking contour lines of the 
(a)

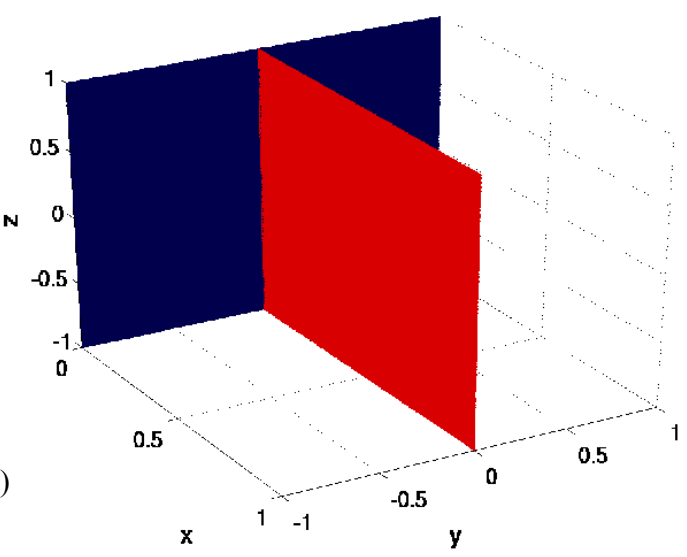

(b)

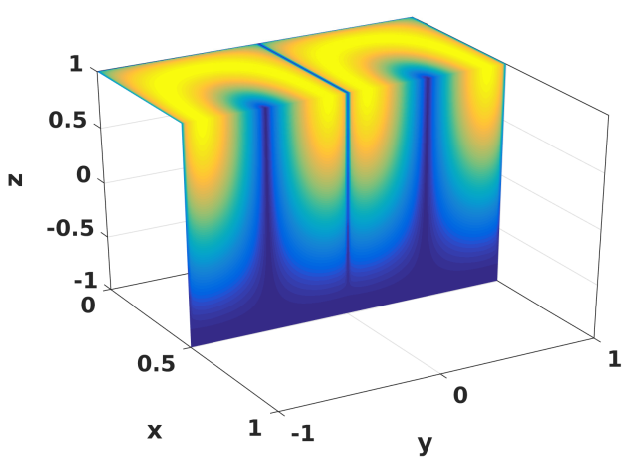

Figure 1. (a) Stable (blue) and unstable (red) manifolds of the normally hyperbolic invariant curve in Eq. (2); (b) representation of the Lagrangian structures at specific slices by means of the $M$ function.

time average of $M$ with tori-like invariant sets. In this manner singular lines in Fig. 1b highlight invariant manifolds and contour lines of converged averages of $M$ highlight invariant tori (see Lopesino et al., 2017).

\section{Dataset and computation of the $M$ function}

\subsection{ERA-Interim Reanalysis dataset}

We use the ERA-Interim Reanalysis dataset produced by European Centre for Medium-Range Weather Forecasts (ECMWF; Simmons et al., 2007). The usefulness of this dataset for the Lagrangian study of atmospheric flows from the dynamical systems perspective is established by the results of several previous studies. For instance, de la Cámara et al. (2013) applied Lagrangian descriptors to study the structure of the SPV during the southern spring of 2005, to support the interpretation of several features found in the trajectories of superpressure balloons released from Antarctica by the VORCORE project (Rabier et al., 2010). ERA-Interim covers the period from 1979 to the present day (Dee et al., 2011), and can be downloaded from http://apps.ecmwf.int/ datasets/data/interim-full-daily/levtype $=\mathrm{sfc} /$.
From the ERA-Interim dataset we extract the horizontal wind velocity components $u$ and $v$, vertical velocity $\omega(\mathrm{d} P / \mathrm{d} t)$, temperature, specific humidity, potential vorticity, surface pressure, and the geopotential field. In the version of the dataset that we selected for the present study, these physical variables are available four times daily (00:00, 06:00, 12:00, 18:00 UTC) with a horizontal resolution of $0.75^{\circ} \times 0.75^{\circ}$ in longitude and latitude. The velocity fields, temperature and specific humidity extracted correspond to the 60 hybrid-sigma levels of the model component of the reanalysis system (from the Earth's surface to the $0.1 \mathrm{hPa}$ level). We also take from the dataset potential vorticity at 15 levels of potential temperature $(265,275,285,300,315$, $330,350,370,395,430,475,530,600,700$, and 850; K) and geopotential at field pressure levels $(1,2,3,5,7,10,20,30$, $50,70,100$ to 250 by 25,300 to 750 by 50,775 to 1000 by $25 ; \mathrm{hPa})$.

\subsection{Computation of the $M$ function}

The procedure to obtain the $M$ function in (lat, lon, height) coordinates from the data described in the previous section consists of the following steps.

- Step 1. The data are downloaded from ERA-Interim in . grib format and on a monthly basis.

- Step 2. The data files are converted from . grib to . nc format with the Climate Data Operator (CDO) software (available at https://code.zmaw.de/projects/cdo). This is done with the copy command, setting as arguments - $t$ ecmwf to indicate that the data are from ERA-Interim and $-f$ nc to specify that the output complies with NetCDF.

- Step 3. The vertical velocity $\omega$, the temperature and the specific humidity are concatenated using the CDO command merge to provide the input to the function vertwind to compute the vertical velocity $w$ in metres per second $\left(\mathrm{m} \mathrm{s}^{-1}\right)$.

- Step 4 . Then, the 3-D velocity $(u, v, w)$ and 2-D surface pressure data are also concatenated to produce the input required in the next step. using the merge CDO command.

- Step 5. The data are converted from sigma levels to the height levels specified for the analysis, and required data are produced by interpolation, with all velocity components expressed in metres per second $\left(\mathrm{m} \mathrm{s}^{-1}\right)$. This is done using the CDO command $\mathrm{ml} 2 \mathrm{hlx}$ applied to the NetCDF files. The resulting 3-D velocity field has a horizontal resolution of $0.75^{\circ} \times 0.75^{\circ}$ in longitude and latitude, with 80 height levels ranging from 0 to $47600 \mathrm{~m}$ at $600 \mathrm{~m}$ intervals. Each wind data variable is stored in separate files by day using the selvar and selday commands. This procedure avoids the handling of very 
large data files when computing particle trajectories, which requires interpolation in time and space.

- Step 6. To avoid issues at the pole in the calculation of trajectories from equations expressed in spherical coordinates, the velocities are written in Cartesian coordinates from the velocity data available in spherical coordinates (see de la Cámara et al., 2012); the velocity components in Cartesian coordinates are given by

$\left\{\begin{array}{l}v_{x}=w \cos \lambda \cos \phi-u \sin \lambda-v \cos \lambda \sin \phi, \\ v_{y}=w \sin \lambda \cos \phi+u \cos \lambda-v \sin \lambda \sin \phi, \\ v_{z}=w \sin \phi+v \cos \phi,\end{array}\right.$

where $u, v$, and $w$ are the zonal, meridional, and vertical velocity components, respectively, $\lambda$ is longitude, and $\phi$ is latitude.

- Step 7. The trajectories are calculated in a Cartesian coordinate system, which are obtained by solving

$\left\{\begin{array}{l}\frac{\mathrm{d} x}{\mathrm{~d} t}=v_{x}(\lambda, \phi, h, t) \\ \frac{\mathrm{d} y}{\mathrm{~d} t}=v_{y}(\lambda, \phi, h, t) . \\ \frac{\mathrm{d} z}{\mathrm{~d} t}=v_{z}(\lambda, \phi, h, t)\end{array}\right.$

To integrate the system (7), the data interpolation of the fields $v_{x}, v_{y}, v_{z}$ is carried out by interpolating $u, v, w$ in spherical coordinates, since the post-processed data files from ERA-Interim are expressed in this way. In order to do so, we have used the griddedinterpolant function provided by the MATLAB $\odot$ software, which generates an object that can be saved into memory and evaluated at any point of interest in the trajectory at a later time. This allows for substantial saving in computational time at every integration step. Moreover, when applying this function to interpolate the dataset, we specify that a cubic interpolation is used in space and also in time. The system (7) is integrated using a CashKarp Runge-Kutta scheme (Press et al., 1992) with a time step of $1 \mathrm{~h}$. The condition $w=0$ is imposed at $h=0$ in order to constrain trajectories in the vertical. Important computational savings are also achieved by integrating simultaneously the whole mesh grid of initial conditions by using a matrix formulation. Additionally, the computational time is reduced by running the MATLAB $\odot$ software with options requiring the use of multiple cores.

- Step 8. The $M$ function is obtained by approximating the integral in Eq. (5) by the sum of the lengths (in the Euclidean space) of the segments linking the position of the integrated particle trajectory at two successive time steps. As $\tau$ increases, a richer Lagrangian history is incorporated into $M$, and a more complex and detailed dynamical description is obtained.

\section{Benchmarks}

To benchmark the procedure described in the previous section, we compare the Lagrangian outputs obtained from the full 3-D scenario at constant heights (spherical shells) with those obtained from the 2-D scenario for potential temperature surfaces that are approximately at the same height. Figure 2 compares, for different $\tau$ values, the evaluation of the $M$ function for a 2-D integration in the $850 \mathrm{~K}$ isentropic surface with that obtained on a spherical shell at $31.3 \mathrm{~km}$ height, which approximately corresponds to the $850 \mathrm{~K}$ surface. For $\tau=5,10$ days, the figure highlights well-defined structures (de la Cámara et al., 2012) that are very similar in both cases. These consist of a large circulating coherent vortex in yellow, representing high values of $M$ that are related to particles exhibiting large displacements and blueish zones corresponding to calmer regions. Lobes eroding the outer part of the vortex are clearly identified by coloured filaments. Also, crossings of contours of $M$ highlighting hyperbolic trajectories are noticed along longitudes $15^{\circ} \mathrm{W}$ and $165^{\circ} \mathrm{E}$. These results confirm that in the upper stratosphere the potential temperature surface and the spherical shell are almost identical.

Increasing the $\tau$ values to 20 or 30 days adds more Lagrangian detail to the figures, which thus contain long-term transport issues reflected in very thin and long filamentous structures. However, the figures at constant potential temperature and constant height are still very similar and contain the gross structure already observed at smaller $\tau$. Differences are restricted to those filamentous structures which are difficult to follow and to compare. Given that long-term transport issues are difficult to interpret and that we do not require them to describe the phenomena of our interest which occur in time intervals varying from hours to 10 days, we fix in this range the selected $\tau$ values for this article.

Comparisons of transport between both approaches are rather different, as Fig. 3 confirms. The first row in this figure shows the 2-D calculations for a 2-D integration in the $330 \mathrm{~K}$ isentropic surface for a period of $\tau=5,10$ days, and the second row a 3-D output at the corresponding height $(10 \mathrm{~km})$ for the same integration intervals. The panels on the top row of Fig. 3 (at a constant potential temperature) are quite similar to those in the bottom row (at constant height), although differences are more evident than those in Fig. 2 for the upper stratosphere. At this level constant potential temperature surfaces are not so close to a sphere, but flow structures in the troposphere are deep, i.e. they have a vertical scale of about $10 \mathrm{~km}$, which is larger than the vertical separations between points on the $10 \mathrm{~km}$ high sphere and $330 \mathrm{~K}$ surfaces. Results discussed in the next section will show further evidence of the 2-D-3-D motion transitions.

Figure 4 contrasts the more classical description of events in the middle-upper stratosphere with the one provided by our Lagrangian descriptor. The left-hand column of Fig. 4 shows the geopotential height at $10 \mathrm{mb}$. This field is partic- 
(a)

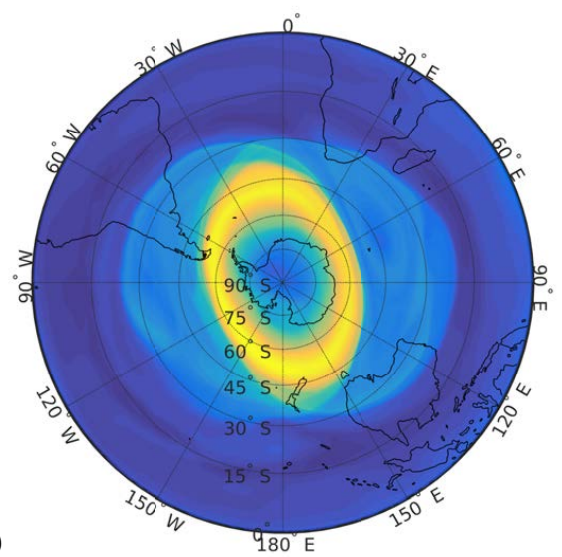

(c)
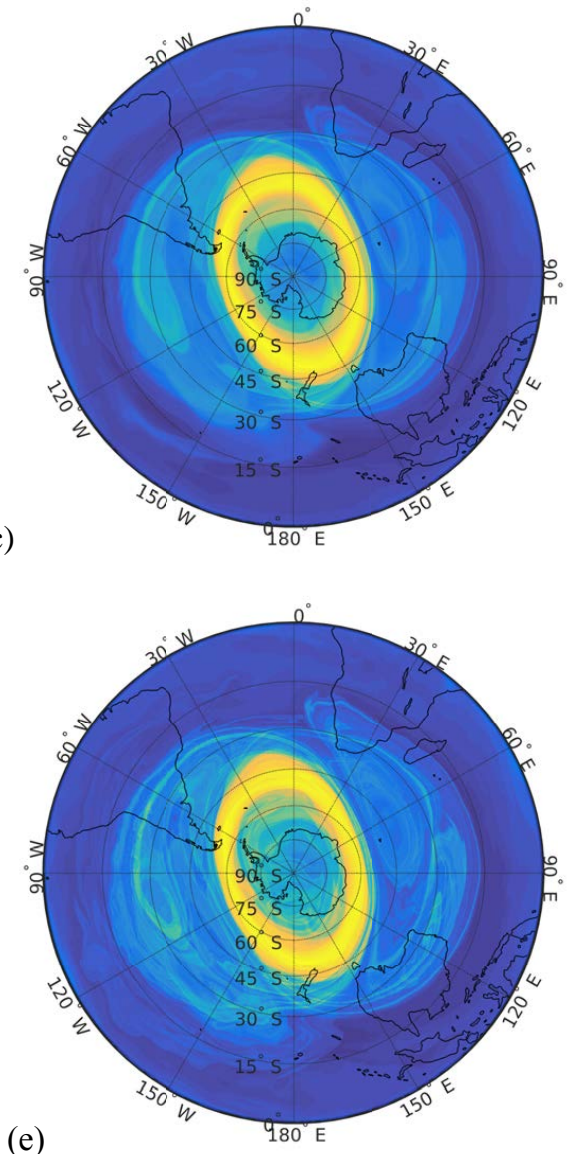

(b)

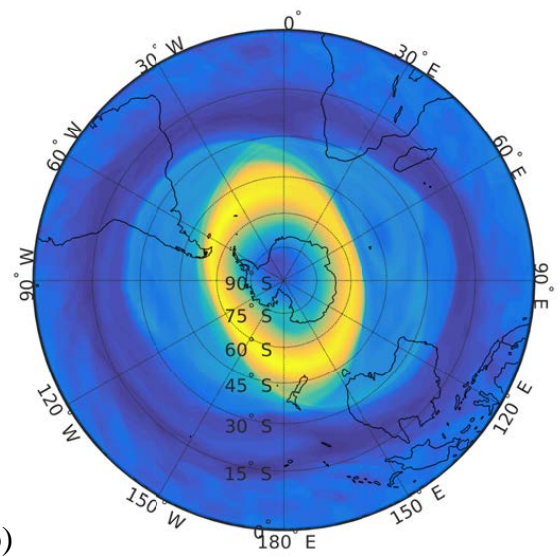

(d)

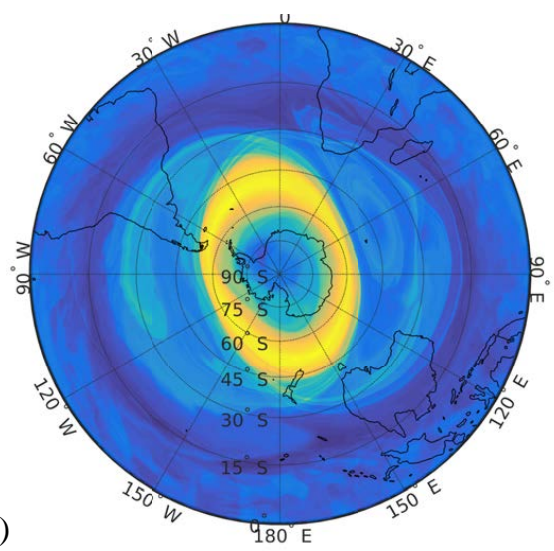

(f)

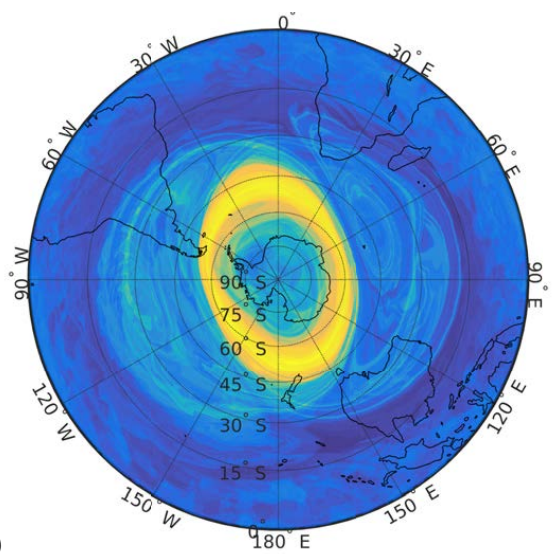

Figure 2. $M$ calculated on 15 August 1979 at 00:00:00 UTC: (a) using a 2-D approach where particle trajectories are constrained to the $850 \mathrm{~K}$ potential temperature level and $\tau=5$ days; (b) $M$ computation for $\tau=5$ days using a full 3-D computation of trajectories, and it is represented at a constant height level of $h=31.3 \mathrm{~km}$ (which corresponds approximately to the $850 \mathrm{~K}$ isentropic surface); (c) the same as panel (a) with $\tau=10$ days; (d) the same as panel (b) with $\tau=10$ days; (e) the same as panel (a) with $\tau=20$ days; (f) the same as panel (b) with $\tau=20$ days.

ularly useful because it is equivalent to the velocity streamfunction at the corresponding pressure level in the extratropics (see García-Garrido et al., 2017). This field provides a purely Eulerian description of the flow; however, in the timedependent case this point of view is limited as it does not address issues regarding the fate of particle trajectories. The middle column shows the picture provided by the potential vorticity at the $850 \mathrm{~K}$ equivalent temperature (isentropic) surface. This field is conserved along particle trajectories; thus, the image is Lagrangian in the sense that we observe the 
(a)

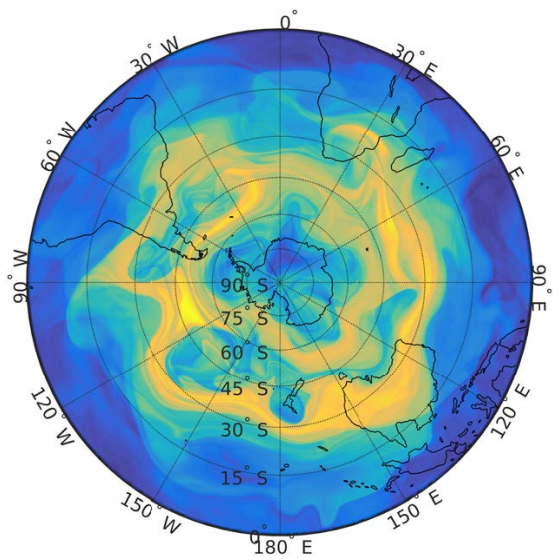

(c)

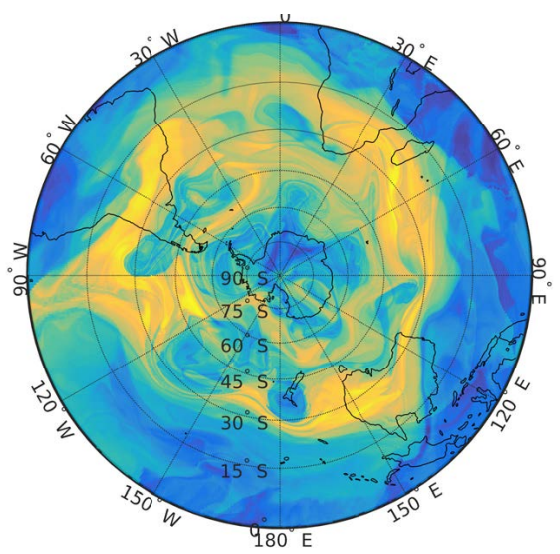

(b)

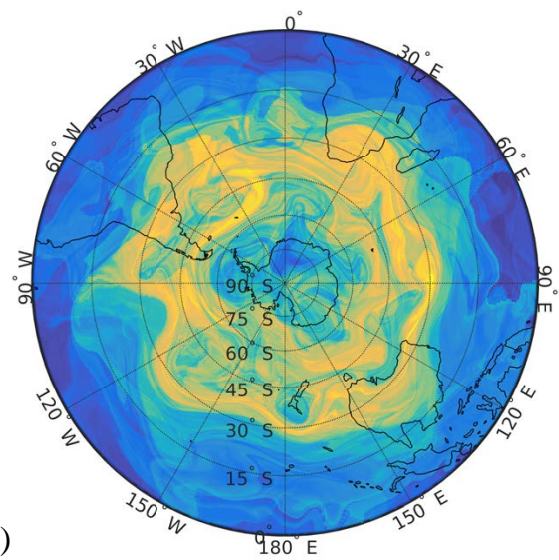

(d)

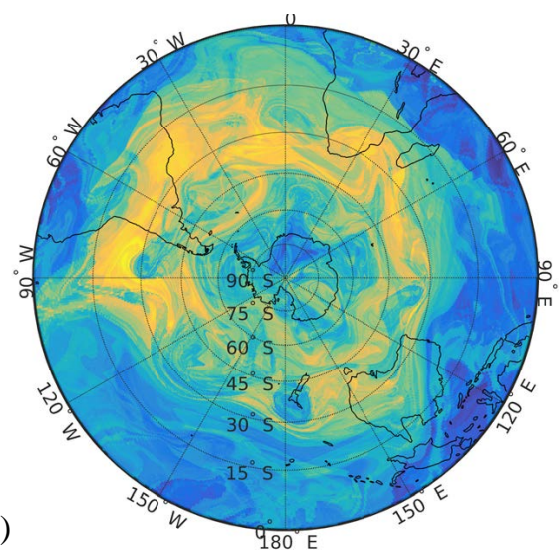

Figure 3. $M$ calculated on 15 August 1979 at 00:00:00 UTC: (a) using a 2-D approach where particle trajectories are constrained to the $330 \mathrm{~K}$ potential temperature level and $\tau=5$ days; (b) the same with $\tau=10$ days; (c) $M$ calculated for $\tau=5$ days using a 3-D computation of trajectories and represented at a constant height level of $h=10 \mathrm{~km}$ (which corresponds approximately to the 330K isentropic surface); (d) the same with $\tau=10$ days.

field of a purely advected quantity. The right-hand column shows the $M$ function at the surface $z=31.3 \mathrm{~km}$ obtained from particle trajectories in full 3-D calculations. Similarly to the second column, the displayed information is Lagrangian, but here we obtain more fundamental information in this regard. This figure provides feedback for characterizing the time evolution of any purely advected scalar field, while the previous one displays just the realization of one particular initial datum. More specifically, the third column highlights the position and evolution of two hyperbolic points in the outer part of the vortex, as well as the vortex itself. As discussed by García-Garrido et al. (2017), hyperbolic points are responsible for filamentation processes. Whether or not these filaments are eventually observed depends on the distribution of the scalar field. For instance, if the scalar field is completely uniform in the whole domain, then its time evolution will show nothing about the features highlighted by $M$. How the features of the $M$ field are visible in a scalar field depends on how the initial distribution of the advected field is with respect to the features of $M$. Figure 5 illustrates these facts in a very simple example. Figure 5a highlights the Lagrangian skeleton as obtained for the stationary cat eyes, i.e. $\dot{x}=y$, $\dot{y}=0.5 \sin x$. The hyperbolic fixed point at the origin and its stable and unstable manifolds are clearly visible. Also, elliptic fixed points at positions $(-\pi, 0)$ and $(\pi, 0)$ are visible. Figure $5 \mathrm{~b}$ shows a set of initial scalar fields and $\mathrm{c}$ shows the evolution of the three patches: the one in cyan shows filamentation, the other staying coherent (in blue) and the third done showing a tongue formation (in red) similar to the one observed for the potential vorticity in Fig. 4. Colours are chosen to highlight the analogous features visible in Fig. 4. Clearly Fig. 5b and c show time-dependent patterns; however, the Lagrangian skeleton shown in Fig. 5a is stationary.

\section{Three-dimensional Lagrangian structures over Antarctica}

The strong and cyclonic SPV characteristic of the winter circulation above Antarctica has been typically represented in the literature by cross sections such as those in Figs. 2 and 


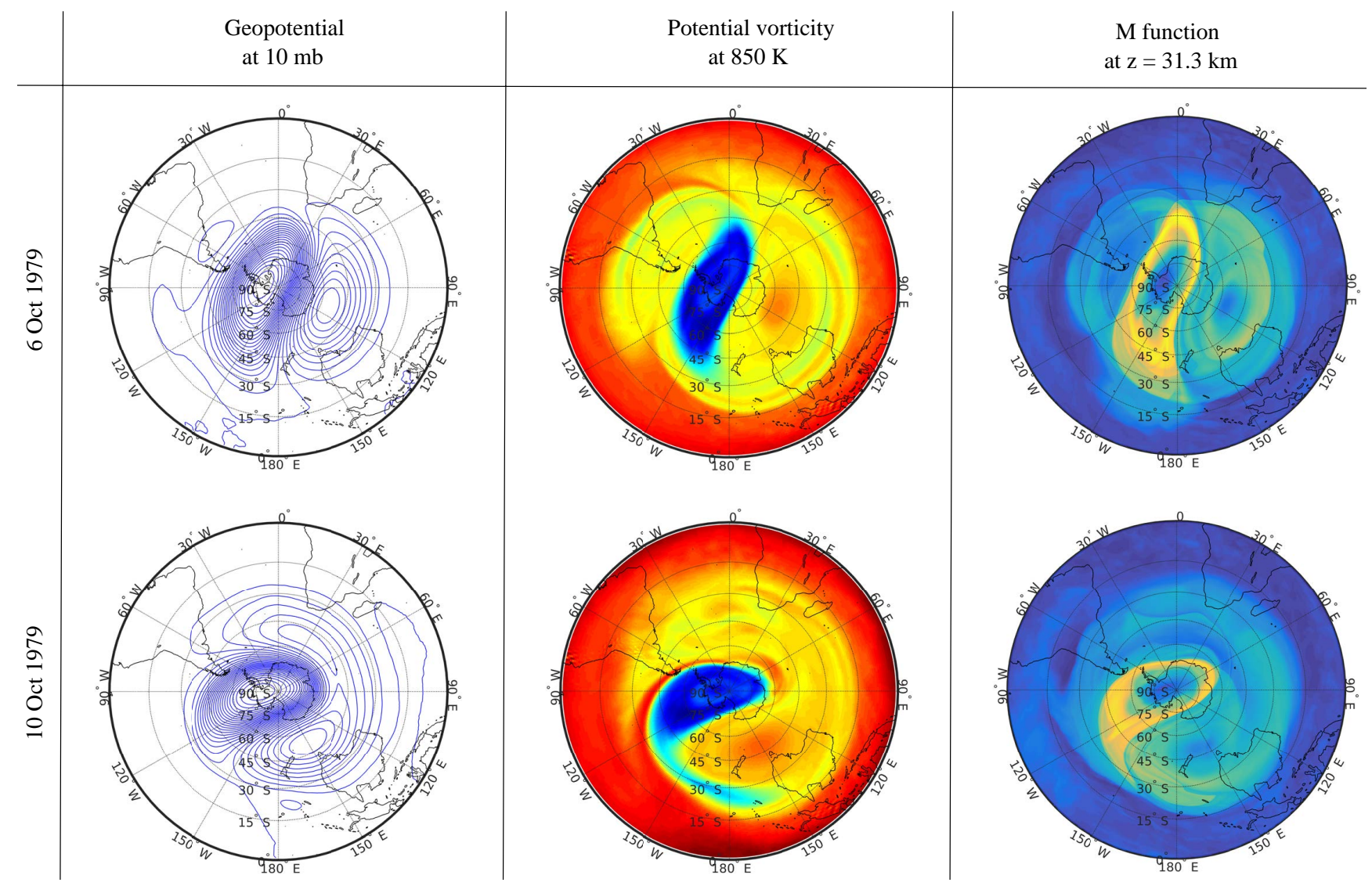

Figure 4. Comparison of geopotential at constant pressure $10 \mathrm{mb}$, potential vorticity at $850 \mathrm{~K}$ and $M$ function at $z=31.3 \mathrm{~km}$ for several days of October 1979.

4. In this section we will improve this representation with figures that are more revealing of the full 3-D description of the circulation. Figure 6 shows for a day in late winter 1979 (15 August) the representation of $M$ obtained for $\tau=5$ for the vertical slice passing through longitudes $90^{\circ} \mathrm{W}$ and $90^{\circ} \mathrm{E}$. An outstanding feature in this representation is the bright yellow colour highlighting a coherent structure. This feature captures the SPV as a tubular structure, similar to those described in Fig. 1b, with walls around $60^{\circ} \mathrm{S}$ and an approximately vertical axis coinciding with that of the Earth and extending from the uppermost level of data down to between 15 and $20 \mathrm{~km}$ in height, i.e. the transition between the troposphere and stratosphere (tropopause). In this case however the average of $M$ does not converge as the flow is aperiodic (see Lopesino et al., 2017), and thus contours of $M$ do not strictly represent invariant sets. Despite this, the setting is analogous to that described for the vortices in the example of Sect. 2. Additionally, the greenish colours that extend equatorward between 15 and $20 \mathrm{~km}$, both in the west and the east, capture the upper tropospheric subtropical westerly jets. Specifically in the west, the greenish colours extend downwards up to the Equator $\left(90^{\circ} \mathrm{W}\right)$, suggesting that the structures involve the entire atmospheric layer. We can also clearly see evidence of the very different dynamical characters of the troposphere and the stratosphere. Whilst the former region is practically dominated by the SPV, the latter shows much finer detail reflected by an intricate line pattern. This tangled pattern is the manifestation of crossings of stable and unstable manifolds, which are associated with strong and fast mixing processes at the lowest atmospheric levels. This image is consistent with and complementary to the projection at $10 \mathrm{~km}$ presented in Fig. $2 \mathrm{c}$ and d and also to the one described next.

The description of the vortex on 15 August 1979 is supplemented by Fig. 7, which shows the $M$ function computed for $\tau=10$ along a vertical slice through latitude $60^{\circ} \mathrm{S}$, where most of the SPV walls are. The intricate structures in the troposphere are also apparent in this figure. In addition, a wavy structure is clearly visible at the boundary between troposphere and stratosphere. In terms of a Fourier decomposition of $M$ at constant height in Fig. 7, we can see the classical pattern of longer wavelengths dominating the field as height increases. At tropopause level, a wavenumber 4 component is clearly visible, while in the upper part of the vortex, a wavenumber 2 is evident (Manney et al., 1991). The vertical propagation of these features across the stratosphere 
(a)

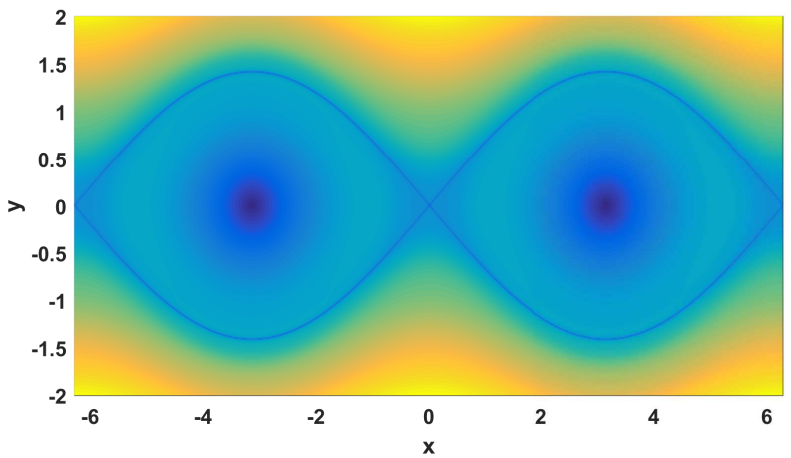

(b)

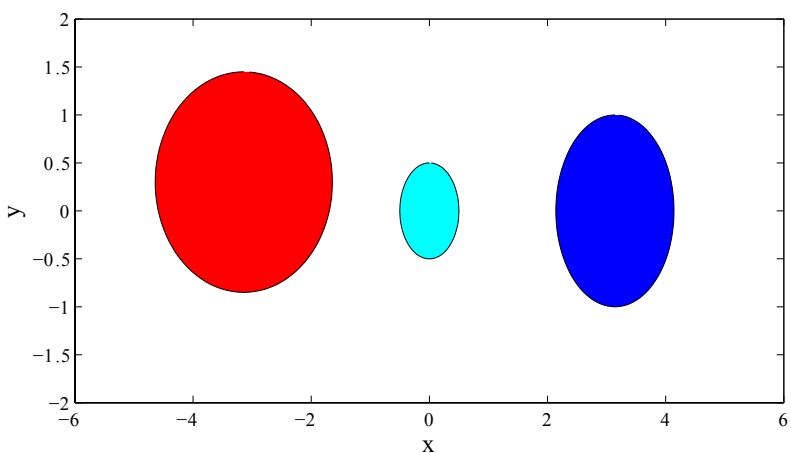

(c)

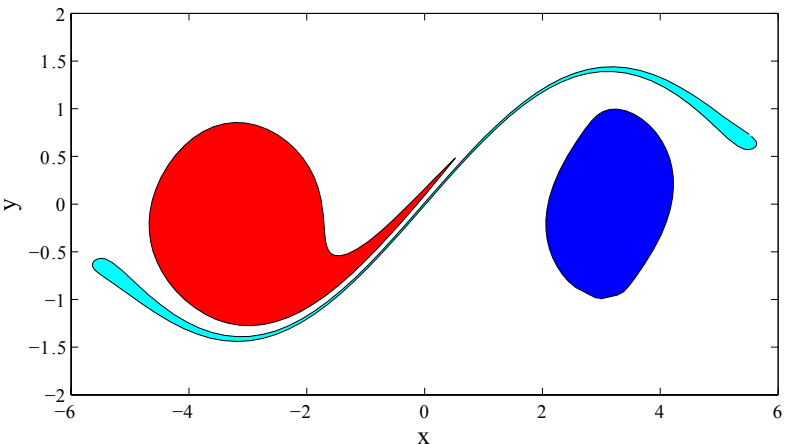

Figure 5. (a) Lagrangian skeleton for the stationary cat eyes; (b) three initial patches of a purely advective field; (c) evolution of the patches at time $t=5.6$.

from 8 to 21 August of 1979 is clearly visible in the attached Movie S1 in the Supplement.

We next focus on the description of the 3-D Lagrangian structures for the period 6-18 October 1979. Figure 8 shows the rapid changes in the SPV that took place in October 1979 as the lower polar stratosphere warmed up strongly during the spring season (Yamazaki and Mechoso, 1985). The figure shows the $M$ function computed for $\tau=5$ along a vertical slice passing through latitude $60^{\circ} \mathrm{S}$ for several October dates. Figure $8 \mathrm{a}, \mathrm{b}, \mathrm{c}$ and $\mathrm{d}$ confirm that Lagrangian structures in the stratosphere become more complex in the warming period. On 18 October no yellow coherent features are visible in the upper stratosphere.

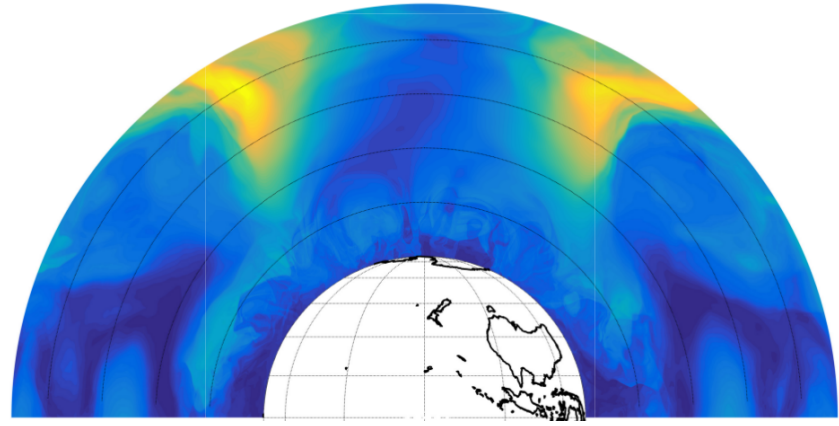

Figure 6. 15 August 1979 00:00:00 UTC. $M$ for $\tau=5$ days displayed along the vertical slice passing through longitudes $90^{\circ} \mathrm{W}$ and $90^{\circ} \mathrm{E}$. The black lines on the stratosphere correspond to heights $10,20,30$ and $40 \mathrm{~km}$ and black lines on the Earth's surface correspond to latitudes $15,30,45,60$, and $75^{\circ} \mathrm{S}$.

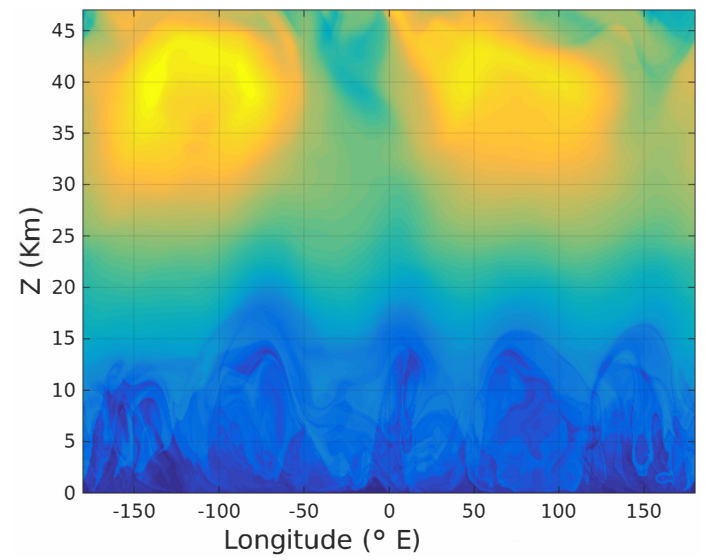

Figure 7. 15 August 1979 00:00:00 UTC. $M$ for $\tau=5$ days displayed along the vertical slice passing through latitude $60^{\circ} \mathrm{S}$.

To help in the interpretation of Fig. 8, Fig. 9 displays horizontal sections of $M$ on 6 October at different heights $z=10,21.2,31.3$ and $40 \mathrm{~km}$ as well as a vertical section along meridians $90^{\circ} \mathrm{E}$ and $90^{\circ} \mathrm{W}$. Figures 8 and 9 show important differences with the winter conditions 2 months earlier, visible in Figs. 6 and 7. The $60^{\circ} \mathrm{S}$ section in Fig. $8 \mathrm{a}$ intersects the cyclonic vortex four times, mostly in the Western Hemisphere. Another deep, anticyclonic vortex appears in the Eastern Hemisphere above $25 \mathrm{~km}$. These cyclonic and anticyclonic vortices are also evident in Fig. $9 \mathrm{a}$ at $z=31.3 \mathrm{~km}$ and $z=40 \mathrm{~km}$, and in Fig. $9 \mathrm{~b}$. In particular in the projection of Fig. 9a at $z=31.3 \mathrm{~km}$, along the longitude $0^{\circ}$, between latitudes 30 and $45^{\circ} \mathrm{S}$, crossing lines marked with a white arrow show the presence of a hyperbolic point, its unstable manifold being the separatrix between the two vortices. The vertical extension of this hyperbolic trajectory is depicted with the red line in Fig. 9c. This figure shows the analogue to the normally hyperbolic invariant curve explained in Sect. 2 with the additional feature that here the flow is time-dependent, while, for simplicity, the example in Sect. 2 
(a) 6 October 1979

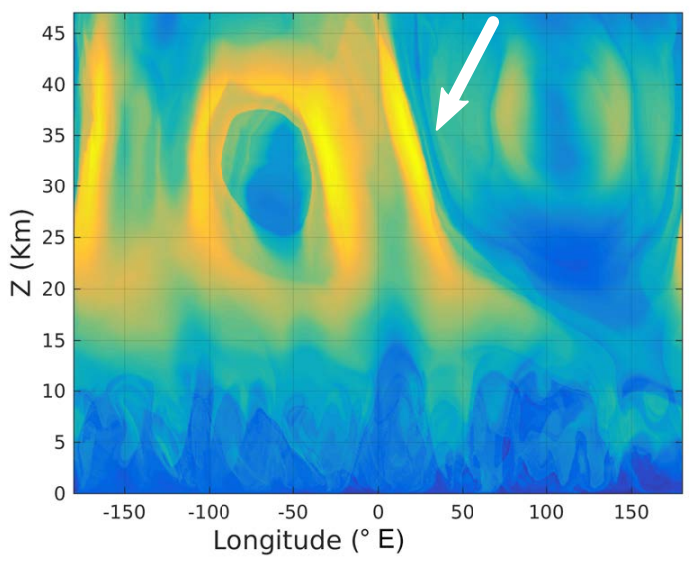

(c) 14 October 1979

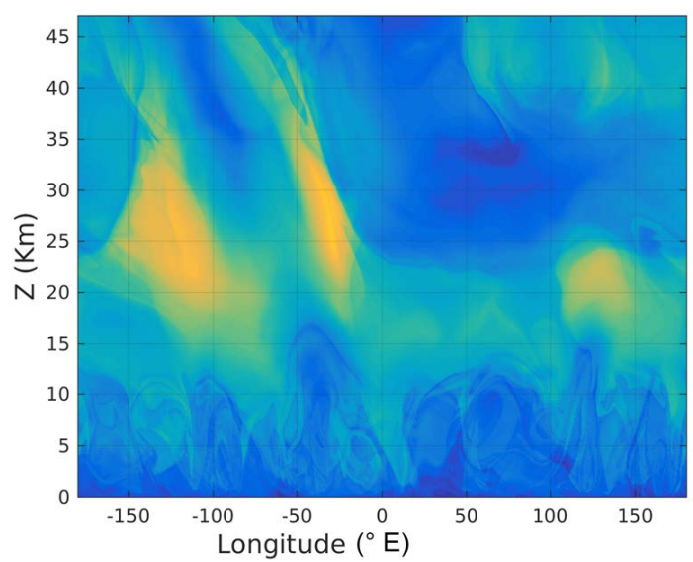

(b) 10 October 1979

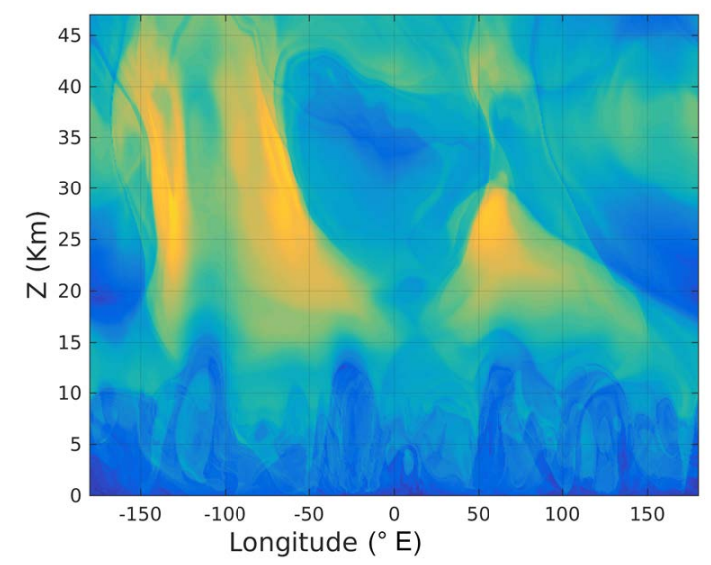

(d) 18 October 1979

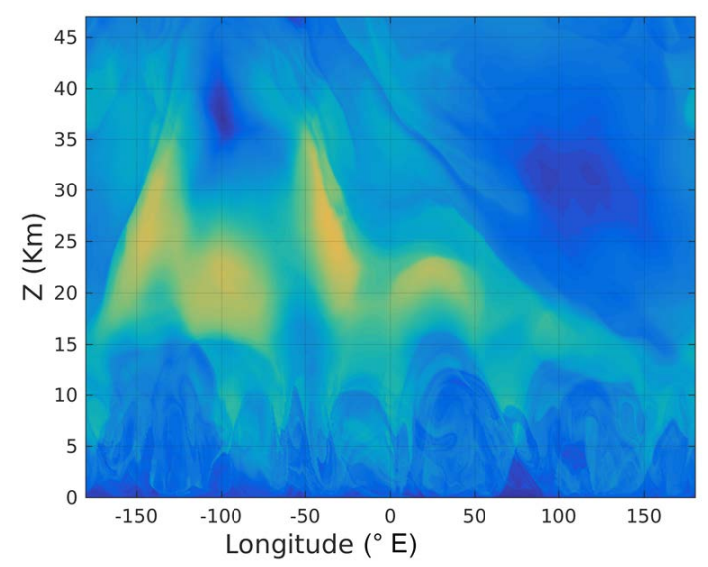

Figure 8. Vertical slices showing $M$ for $\tau=5$ at constant latitude $60^{\circ} \mathrm{S}$ on 4 selected days in October. The colour scale is the same in all figures.

was stationary. Figure $1 \mathrm{~b}$ sketches the corresponding typical configuration for two counterrotating vortex tubes illustrating this description.

The vertically extended unstable manifold of the normally hyperbolic invariant curve that separates the two vortex tubes is captured by $M$ and is visible in Figs. $8 \mathrm{a}$ and $9 \mathrm{~b}$ as a narrow dark blue line in an analogous way to that presented in Fig. 1. The first of these two figures shows it near the edge of the polar vortex, around $45^{\circ} \mathrm{E}$ marked with a white arrow, and for the latter a white arrow also points to the described feature. This manifold structure separates the two counterrotating vortex tubes just described. It acts as a vertical barrier, which is several kilometres deep, as is the case for the unstable manifolds associated with a normally hyperbolic invariant curve formed from hyperbolic trajectories at each $h$ level. Further information on the singular features is given in Fig. 10. The black dots in Fig. 10a and b represent, for
6 October 1979, 00:00:00 UTC, the horizontal and vertical positions of a particle located on the feature indicated by the dark blue line at a height of $31.3 \mathrm{~km}$, longitude $90^{\circ} \mathrm{E}$ and latitude $77.74^{\circ} \mathrm{S}$. The black dots in Fig. 10c and d show the corresponding locations of the same particle $6 \mathrm{~h}$ later. The invariant character of the singular structure is confirmed as the particle remains on it during its evolution, and its unstable character is confirmed by the fact that the particle moves away from the hyperbolic point.

Mechoso and Hartmann (1982) have suggested that the fact that the preferred geographical location (ridge south of Australia) for the development of this anticyclonic vortex in this particular event indicates that the stratospheric circulation is governed to a significant extent from below (see also Quintanar and Mechoso, 1995). This anticyclonic vortex will strengthen and eventually dominate at high levels. In terms of Fourier components, a quasi-stationary wave 1 amplifies 

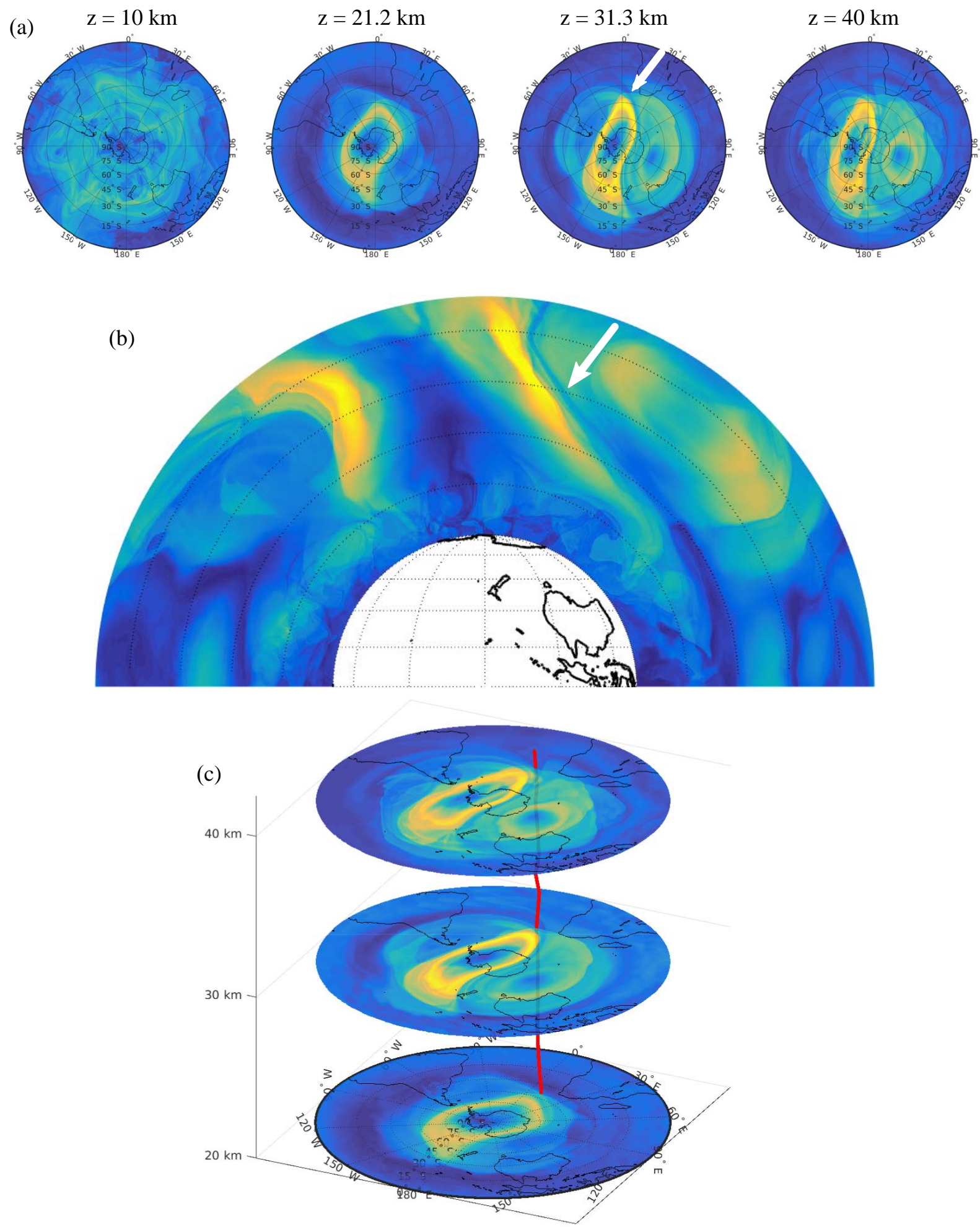

Figure 9. 6 October 1979 00:00:00 UTC. (a) Slices of $M$ at constant heights $z=10,21.2,31.3$ and $40 \mathrm{~km}$ with $\tau=5$; (b) slice of $M$ at constant longitude $\lambda=90^{\circ} \mathrm{W}$ and $\lambda=90^{\circ} \mathrm{E}$. (b) The black lines on the stratosphere correspond to heights $10,20,30$ and $40 \mathrm{~km}$ and black lines on the Earth's surface correspond to latitudes $15,30,45,60$, and $75^{\circ} \mathrm{S}$. (c) Slices of $M$ at constant heights $z=21,30$, and $39 \mathrm{~km}$; the red line indicates the position of the hyperbolic point marked with a white arrow in panel (a) for different heights, i.e it is the normally hyperbolic invariant curve. 
(a)

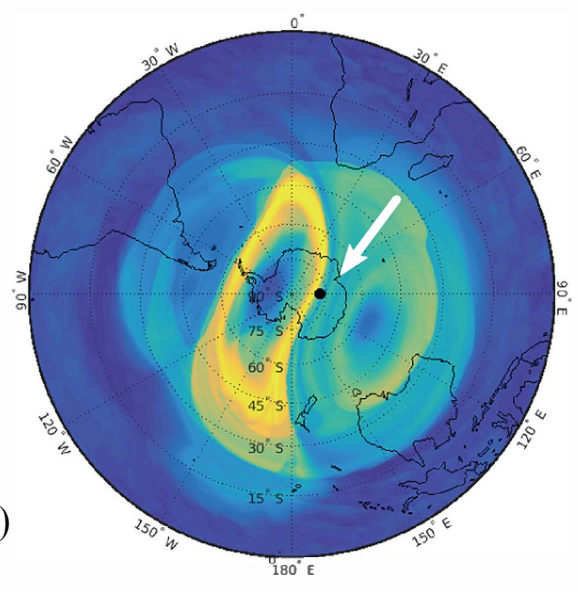

(c)

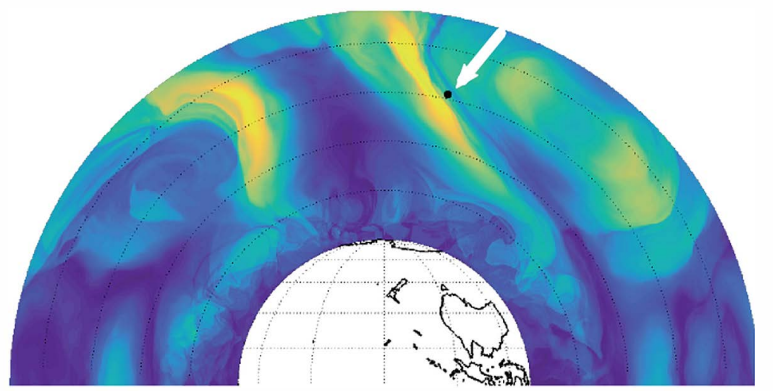

(b)

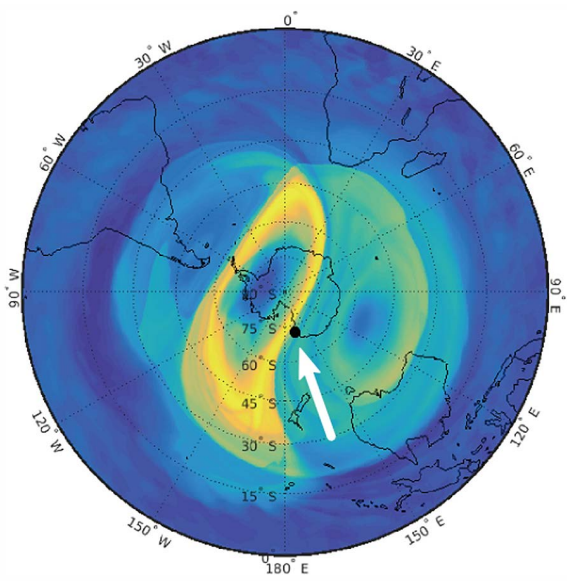

(d)

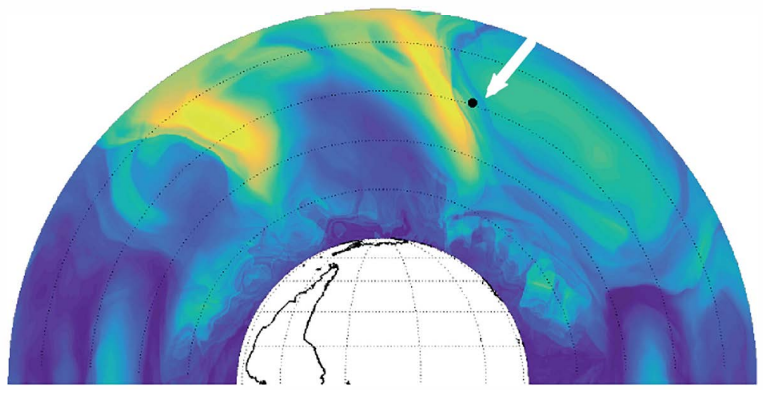

Figure 10. Evaluation of the $M$ function with a black particle on it. (a) The black particle is placed exactly over an invariant manifold on a 2-D slice obtained at a height of $31.3 \mathrm{~km}$ on 6 October 1979, 00:00:00 UTC; (b) the same black particle on the same day and time placed on a 2-D slice obtained at longitude $90^{\circ} \mathrm{E}$; (c) the same black particle $6 \mathrm{~h}$ later on a 2-D slice of $M$ obtained at the corresponding height of the particle at that time; (d) the same black particle $6 \mathrm{~h}$ later on a 2-D slice of $M$ obtained at the corresponding longitude of the particle at that time.

on this date, in conjunction with the displacement of the cyclonic vortex from the polar position.

\section{Conclusions}

In the present paper we discuss the visualization of threedimensional Lagrangian structures in atmospheric flows. Specifically, we have explained mathematical aspects about the Lagrangian geometrical structures to be expected in the atmospheric setting in 3-D and have introduced the concept of normally hyperbolic invariant curves in a specific example which recovers features of those observed in the stratosphere. The algorithm used to represent the 3-D Lagrangian structures is based on the methodology of Lagrangian descriptors (LDs). We have explored the application of the full power of the $M$ function computed with 3-D trajectories, which hitherto had been used in 2-D settings. The consistency of our development has been verified by comparing the 3-D scenario results at a constant height with those obtained from the 2-D scenario in potential temperature surfaces at equivalent heights.

To demonstrate the methodology we have applied it to a numerical dataset describing the flow above Antarctica dur- ing the southern mid-late winter and spring. The dataset was obtained from ERA-Interim Reanalysis data provided by the ECMWF. Our findings show the vertical extension and structure of the stratospheric polar vortex and its evolution. We also characterize, from the Lagrangian point of view, the boundary between the troposphere and the stratosphere. Very complex Lagrangian patterns are identified in the troposphere, which support the presence of strong mixing processes. The "final stratospheric warming" is characterized by the breakdown of the westerly SPV during the transition from winter to summer circulation. Our results confirm that the onset of this process is characterized by an initial decay of the vortex in the upper stratosphere where the circulation weakens, albeit remaining strong at lower heights. We have also captured the anticyclonic circulation that develops during October preferentially above the southern part of Australia. We illustrate the vertical structure of these two counterrotating vortices, and the invariant separatrix that divides them. The particular feature found is several kilometres deep and we demonstrated that fluid parcels remain in this feature during intervals of the order of days. Such features highlight the complexities in the transport of chemical tracers in the stratosphere. 
Data availability. The datasets used in this work are described in Sect. 3, where links are provided to the official websites from which they have been downloaded.

\section{The Supplement related to this article is available online at https://doi.org/10.5194/npg-24-379-2017-supplement.}

Competing interests. The authors declare that they have no conflict of interest.

Special issue statement. This article is part of the special issue "Current perspectives in modelling, monitoring, and predicting geophysical fluid dynamics". It is not part of a conference.

Acknowledgements. Jezabel Curbelo, Víctor José García-Garrido and Ana Maria Mancho are supported by MINECO grant MTM2014-56392-R. Coumba Niang acknowledges Fundacion Mujeres por Africa and ICMAT Severo Ochoa project SEV-20110087 for financial support. Ana Maria Mancho and Coumba Niang are supported by CSIC grant COOPB20265. The research of Stephen Wiggins is supported by ONR grant no. N00014-01-10769. Carlos Roberto Mechoso was supported by U.S. NSF grant AGS-1245069. We also acknowledge support from ONR grant no. N00014-16-1-2492. Thanks are owed to CESGA and ICMAT for computing facilities.

Edited by: Emilio Hernández-García

Reviewed by: two anonymous referees

\section{References}

Aref, H.: Stirring by chaotic advection, J. Fluid Mech., 143, 1-21, 1984.

Bettencourt, J. H., López, C., Hernández-García, E., Montes, I., Sudre, J., Dewitte, B., Paulmier, A., and Garçon, V.: Boundaries of the Peruvian oxygen minimum zone shaped by coherent mesoscale dynamics, Nat. Geosci., 8, 937-940, 2014.

Bowman, K. P.: Large-scale isentropic mixing properties of the Antarctic polar vortex from analyzed winds, J. Geophys. Res., 98, 23013-23027, https://doi.org/10.1029/93JD02599, 1993.

Branicki, M. and Kirwan Jr., A. D.: Stirring: The Eckart paradigm revisited, Int. J. Eng. Sci., 48, 1027-1042, 2010.

Branicki, M. and Wiggins, S.: An adaptive method for computing invariant manifolds in non-autonomous, three-dimensional dynamical systems, Physica D, 238, 1625-1657, 2009.

Branicki, M., Mancho, A. M., and Wiggins, S.: A Lagrangian description of transport associated with a Front-Eddy interaction: application to data from the North-Western Mediterranean Sea, Physica D, 240, 282-304, 2011.

Cartwright, J. H. E., Feingold, M., and Piro, O.: Chaotic adection in three-dimensional unsteady incompressible laminar flow, J. Fluid Mech., 316, 259-284, 1996.
Dee, D. P., Uppala, S. M., Simmons, A. J., Berrisford, P., Poli, P., Kobayashi, S., Andrae, U., Balmaseda, M. A., Balsamo, G., Bauer, P., Bechtold, P., Beljaars, A. C. M., van de Berg, L., Bidlot, J., Bormann, N., Delsol, C., Dragani, R., Fuentes, M., Geer, A. J., Haimberger, L., Healy, S. B., Hersbach, H., Hólm, E. V.,Isaksen, L., Kållberg, P., Köhler, M., Matricardi, M., McNally, A. P., Monge-Sanz, B. M., Morcrette, J.-J., Park, B.-K., Peubey, C., de Rosnay, P., Tavolato, C., Thépaut, J.-N., and Vitart, F.: The ERA-Interim reanalysis: configuration and performance of the data assimilation system, Q. J. Roy. Meteor. Soc., 137, 553-597, https://doi.org/10.1002/qj.828, 2011.

de la Cámara, A., Mancho, A. M., Ide, K., Serrano, E., and Mechoso, C.: Routes of transport across the Antarctic polar vortex in the southern spring., J. Atmos. Sci., 69, 753-767, 2012.

de la Cámara, A., Mechoso, R., Mancho, A. M., Serrano, E., and Ide., K.: Isentropic transport within the Antarctic polar night vortex: Rossby wave breaking evidence and Lagrangian structures, J. Atmos. Sci., 70, 2982-3001, 2013.

d'Ovidio, F., Isern-Fontanet, J., López, C., Hernández-García, E., and García-Ladona, E.: Comparison between Eulerian diagnostics and finite-size Lyapunov exponents computed from altimetry in the Algerian basin, Deep-Sea Res. Pt. I, 56, 15-31, 2009.

du Toit, P. C. and Marsden, J. E.: Horseshoes in hurricanes, J. Fix. Point Theory A., 7, 351-384, 2010.

García-Garrido, V. J., Mancho, A. M., Wiggins, S., and Mendoza, C.: A dynamical systems approach to the surface search for debris associated with the disappearance of flight MH370, Nonlin. Processes Geophys., 22, 701-712, https://doi.org/10.5194/npg22-701-2015, 2015.

García-Garrido, V. J., Ramos, A., Mancho, A. M., Coca, J., and Wiggins, S.: A dynamical systems perspective for a real-time response to a marine oil spill, Mar. Pollut. Bull., 112, 201-210, https://doi.org/10.1016/j.marpolbul.2016.08.018, 2016.

García-Garrido, V. J., Curbelo, J., Mechoso, C. R., Mancho, A M., and Wiggins, S.: A simple kinematic model for the Lagrangian description of relevant nonlinear processes in the stratospheric polar vortex, Nonlin. Processes Geophys., 24, 265-278, https://doi.org/10.5194/npg-24-265-2017, 2017.

Guha, A., Mechoso, C. R., Konor, C. S., and Heikes, R. P.: Modeling Rossby Wave Breaking in the Southern Spring Stratosphere, J. Atmos. Sci., 73, 393-406, 2016.

Haller, G. and Yuan, G.: Lagrangian coherent structures and mixing in two-dimensional turbulence, Physica D, 147, 352-370, 2000.

Joseph, B. and Legras, B.: Relation between Kinematic Boundaries, Stirring, and Barriers for the Antarctic Polar Vortex, J. Atmos. Sci., 59, 1198-1212, 2002.

Lekien, F. and Ross, S. D.: The computation of finitetime Lyapunov exponents on unstructured meshes and for non-Euclidean manifolds, Chaos, 20, 017505 , https://doi.org/10.1063/1.3278516, 2010.

Lopesino, C., Balibrea-Iniesta, F., García-Garrido, V. J., Wiggins, S., and Mancho, A. M.: A theoretical framework for lagrangian descriptors, Int. J. Bifurcat. Chaos, 27, 1730001, https://doi.org/10.1142/S0218127417300014, 2017.

Madrid, J. A. J. and Mancho, A. M.: Distinguished trajectories in time dependent vector fields, Chaos, 19, 013111, https://doi.org/10.1063/1.3056050, 2009.

Mancho, A. M., Hernández-García, E., Small, D., Wiggins, S., and Fernández, V.: Lagrangian transport through an ocean front in 
the North-Western Mediterranean Sea, J. Phys. Oceanogr., 38, 1222-1237, 2006.

Mancho, A. M., Wiggins, S., Curbelo, J., and Mendoza, C.: Lagrangian descriptors: A Method for Revealing Phase Space Structures of General Time Dependent Dynamical Systems, Commun. Nonlinear Sci., 18, 3530-3557, 2013.

Manney, G. L. and Lawrence, Z. D.: The major stratospheric final warming in 2016: dispersal of vortex air and termination of Arctic chemical ozone loss, Atmos. Chem. Phys., 16, 15371-15396, https://doi.org/10.5194/acp-16-15371-2016, 2016.

Manney, G. L., Farrara, J. D., and Mechoso, C. R.: The behavior of wave 2 in the southern hemisphere stratosphere during late winter and early spring., J. Atmos. Sci., 48, 976-998, 1991.

Mechoso, C. R. and Hartmann, D. L.: An Observational Study of Traveling Planetary Waves in the Southern Hemisphere, J. Atmos. Sci., 39, 1921-1935, https://doi.org/10.1175/15200469(1982)039<1921:AOSOTP>2.0.CO;2, 1982.

Mechoso, C. R., O’Neill, A., Pope, V. D., and Farrara, J. D.: A study of the stratospheric final warming of 1982 in the Southern Hemisphere, Q. J. Roy. Meteor. Soc., 114, 1365-1384, 1988.

Mendoza, C. and Mancho, A. M.: The hidden geometry of ocean flows, Phys. Rev. Lett., 105, 038501, https://doi.org/10.1103/PhysRevLett.105.038501, 2010.

Mendoza, C., Mancho, A. M., and Wiggins, S.: Lagrangian descriptors and the assessment of the predictive capacity of oceanic data sets, Nonlin. Processes Geophys., 21, 677-689, https://doi.org/10.5194/npg-21-677-2014, 2014.

Mezić, I. and Wiggins, S.: On the integrability and perturbation of three-dimensional fluid flows with symmetry, J. Nonlinear Sci., 4, 157-194, 1994.

Mezic, I. and Wiggins, S.: A method for visualization of invariant sets of dynamical systems based on the ergodic partition, Chaos, 9, 213-218, 1999.

Moharana, N. R., Speetjens, M. F. M., Trieling, R. R., and Clercx, H. J. H.: Three-dimensional Lagrangian transport phenomena in unsteady laminar flows driven by a rotating sphere, Phys. Fluids, 25, 093602, https://doi.org/10.1063/1.4819901, 2013.

Pouransari, Z., Speetjens, M. F. M., and Clercx, H. J. H.: Formation of coherent structures by fluid inertia in three-dimensional laminar flows, J. Fluid Mech., 654, 5-34, 2010.

Press, W. H., Teukolsky, S. A., Vetterling, W. T., and Flannery, B. P.: Numerical Recipes in C: The Art of Scientific Computing, Cambridge University Press, New York, NY, USA, 1992.

Quintanar, A. I. and Mechoso, C. R.: Quasi-stationary waves in the Southern Hemisphere. Part I: Observational data, J. Climate, 4, 2659-2672, 1995.
Rabier, F., Bouchard, A., Brun, E., Doerenbecher, A., Guedj, S., Guidard, V., Karbou, F., Peuch, V., El Amraoui, L., Puech, D., Genthon, C., Picard, G., Town, M., Hertzog, A., Vial, F., Cocquerez, P., Cohn, S. A., Hock, T., Fox, J., Cole, H., Parsons, D., Powers, J., Romberg, K., VanAndel, J., Deshler, T., Mercer, J., Haase, J. S., Avallone, L., Kalnajs, L., Mechoso, C. R., Tangborn, A., Pellegrini, A., Frenot, Y., Thépaut, J., McNally, A., Balsamo, G., and Steinle, P.: The Concordiasi Project in Antarctica, B. Am. Meteorol. Soc., 91, 69-86, https://doi.org/10.1175/2009BAMS2764.1, 2010.

Rempel, E. L., Chian, A. C.-L., Brandenburg, A., Munuz, P. R., and Shadden, S. C.: Coherent structures and the saturation of a nonlinear dynamo, J. Fluid Mech., 729, 309-329, 2013.

Rutherford, B. and Dangelmayr, G.: A three-dimensional Lagrangain hurricane eyewall computation, Q. J. Roy. Meteor. Soc., 136, 1931-1944, 2010.

Rutherford, B., Dangelmayr, G., and Montgomery, M. T.: Lagrangian coherent structures in tropical cyclone intensification, Atmos. Chem. Phys., 12, 5483-5507, https://doi.org/10.5194/acp-12-5483-2012, 2012.

Rypina, I. I., Pratt, L. J., Wang, P., Özgökmen, T. M., and Mezic, I.: Resonanace phenomena in a time-dependent, threedimensional model of an idealized eddy, Chaos, 25, 087401, https://doi.org/10.1063/1.4916086, 2015.

Shadden, S. C., Lekien, F., and Marsden, J. E.: Definition and properties of Lagrangian Coherent Structures from finite-time Lyapunov exponents in two-dimensional aperiodic flows, Physica D, 212, 271-304, 2005.

Simmons, A., Uppala, S., Dee, D., and Kobayashi, S.: ERA-Interim: New ECMWF reanalysis products from 1989 onwards, ECMWF Newsletter, 110, 25-35, 2007.

Smith, M. L. and McDonald, A. J.: A quantitative measure of polar vortex strength using the function M, J. Gephys. Res.-Atmos., 119, 5966-5985, 2014.

Wiggins, S.: Global bifurcations and chaos: analytical methods, vol. 73, Springer Verlag, New York, USA, 1988.

Wiggins, S.: Coherent structures and chaotic advection in three dimensions, J. Fluid Mech., 654, 1-4, https://doi.org/10.1017/S0022112010002569, 2010.

Yamazaki, K. and Mechoso, C. R.: Observations of the Final Warming in the Stratosphere of the Southern Hemisphere during 1979, J. Atmos. Sci., 42, 1198-1205, 1985. 